\title{
Validation study of perfusion parameter in hypervascular hepatocellular carcinoma and focal nodular hyperplasia using dynamic susceptibility magnetic resonance imaging with super- paramagnetic iron oxide: comparison with single level dynamic CT arteriography
}

\author{
Kazuhiro Saito ${ }^{1}$, Joseph Ledsam ${ }^{2}$, Steven Sourbron ${ }^{2}$, Yoichi Araki ${ }^{1}$ \\ ${ }^{1}$ Department of Radiology, Tokyo Medical University, Shinjuku-ku, Tokyo, Japan; ${ }^{2}$ Division of Biomedical Imaging, Leeds University, Leeds, UK \\ Correspondence to: Kazuhiro Saito. Department of Radiology, Tokyo Medical University, 6-7-1, Nishi-Shinjuku, Shinjuku-ku, Tokyo 1600023, Japan. \\ Email: saito-k@tokyo-med.ac.jp.
}

Background: Dynamic susceptibility contrast MR imaging (DSC-MRI) offers direct evaluation of neo-
vascularity. Ferucarbotran does not accumulate in the interstitial space, instead remaining in the intravascular
space during early phase imaging. We investigate tracer kinetic analysis with DSC-MRI with ferucarbotran
and single level CT during hepatic arteriography (SL-CTHA) in assessment of hypervascular hepatocellular
lesions and evaluate the usefulness of DSC-MRI with ferucarbotran. Methods: Six patients having hypervascular hepatocellular carcinoma (HCC) and 3 patients having focal nodular hyperplasia $(\mathrm{FNH})$ were included in the study. SL-CTHA was performed with the infusion of $3 \mathrm{~mL}$ of contrast media at a rate of $1 \mathrm{~mL} / \mathrm{s}$ and scanned at a rate of 0.8 second per rotation. DSCMRI was acquired with the echo-planar method at $1.5 \mathrm{~T}$ system. A total dose of $1.4 \mathrm{~mL}(0.5 \mathrm{~mol} \mathrm{Fe} / \mathrm{L})$ of ferucarbotran was used. Ferucarbotran was injected at a rate of $2 \mathrm{~mL} / \mathrm{s}$ with $40 \mathrm{~mL}$ of physiological saline. Imaging was obtained at a temporal resolution of 1.2 or 0.46 seconds in 5 and 4 patients, respectively. For both CT and MRI modalities, a model-free analysis method was used to derive region of interest-based perfusion parameters. Plasma flow, distribution volume (DV) of contrast agent and estimated mean transit time (EMTT) were estimated.

Results: A strong correlation was obtained with plasma flow ( $\mathrm{r}=0.8231, \mathrm{P}=0.0064)$ between DSC-MRI and SL-CTHA. No significant correlation was obtained for DV and EMTT between DSC-MRI and SL-CTHA. All perfusion parameters showed no significant difference between SL-CTHA and DSC-MRI in FNH. On the other hand, in HCC, DV and EMTT showed significant differences ( $\mathrm{P}=0.046$ and 0.046), and plasma flow showed no significant difference between DSC-MRI and SL-CTHA.

Conclusions: This pilot study demonstrates the possibility of quantitative analysis of liver tumor using superparamagnetic iron oxide (SPIO)-based agent and highlights the potential for SPIO-based agent in more precisely assessing the perfusion characteristic of hypervascular liver tumors than by using extracellular contrast media.

Keywords: Tracer kinetic modeling; dynamic susceptibility contrast MR imaging (DSC-MRI); dynamic contrast enhanced MRI (DCE-MRI); liver; hepatocellular carcinoma (HCC)

Submitted Aug 02, 2019. Accepted for publication Apr 16, 2020.

doi: 10.21037/qims-18-233

View this article at: http://dx.doi.org/10.21037/qims-18-233 


\section{Introduction}

Magnetic resonance imaging (MRI) is a useful diagnostic tool for investigation of hepatic lesions such as hepatocellular carcinoma (HCC) and focal nodular hyperplasia (FNH), especially after development of liver specific contrast agents. MRI has the advantage of limited radiation exposure; it is feasible to acquire multiple repeat examinations to assess therapeutic outcomes and/or clinical observation. Assessment of tissue perfusion is possible by dynamic contrast enhanced MRI (DCE-MRI) using liver specific contrast agent or non-specific extracellular contrast media (1). The perfusion parameters produced new mechanisms for tumor diagnosis, evaluation of therapeutic outcome and prediction of therapeutic efficacy $(2,3)$. CT may also be used for perfusion studies, and its methodology is well established (4). CT is suitable for evaluation of perfusion as the Hounsfield units used to construct CT images change proportionally with contrast media concentration; conversely the $\mathrm{T} 2^{*}$ effect with high concentration of contrast media MRI is a disadvantage.

Both HCC and FNH show hypervascular characteristics on diagnostic imaging. Typically, imaging findings for overt HCC is enhancement in the arterial phase and washout in the portal or equilibrium phase, and a capsule-like structure around the tumor $(5,6)$. FNH is usually lobulated, well circumscribed and has a central stellate scar with radiating fibrous septa. In cases with completely typical findings, the diagnosis of both entities is relatively easy. But in cases that do not have completely typical findings, the diagnosis can be difficult $(7,8)$.

Dynamic susceptibility contrast MR imaging (DSCMRI) with superparamagnetic iron oxide (SPIO) offers improvements in diagnostic measurements of blood volume over both CT and conventional dynamic MRI (9). DSCMRI offers direct evaluation of neo-vascularity. SPIO contrast media does not accumulate in the interstitial space, instead remaining in the intravascular space during early phase imaging. Consequently, SPIO can depict tumor vessels. Although reports have been published with DSCMRI using SPIO in cerebral infarction and brain tumor in animal studies $(10,11)$ and qualitative analysis in this area has been shown possible $(12,13)$, human abdominal imaging studies have been limited by respiratory motion and susceptibility artifact. Dynamic MRI imaging with SPIO contrast agents also offers safety benefits to patients. Unlike CT imaging, DSC-MRI avoids the risks of ionizing radiation, and SPIO contrast agents can be administered safely to patients with asthma and renal dysfunction.

The efficacy of SPIO DSC-MRI in detection of hepatic lesions has been shown comparable to CT in previous work (13). Contrast enhanced studies offer benefits in clinical detection and functional analysis of lesions. The consistency of perfusion imaging in DSC-MRI using SPIO with single level CT during hepatic arteriography (SLCTHA) has been shown in a previous work (12). The perfusion study in SL-CTHA provided a clear time density curve which were derived from the selective contrast media injection from hepatic artery (14). This method can evaluate hepatic arterial flow exclusively without the influence of portal blood flow.

The present study further investigates tracer kinetic analysis with DSC-MRI with SPIO and SL-CTHA in the assessment of hypervascular hepatocellular lesions.

\section{Methods}

This retrospective study was institutional review board approved and data was collected with implied consent.

\section{Subjects}

The subjects were selected from imaging data acquired between November 2006 and July 2008. Subjects were included in the previous qualitative study (12). This study was re-evaluated quantitatively. The inclusion criteria were as follows: both dynamic MRI images with SPIO contrast media and SL-CTHA had been performed within an interval of less than four weeks and full digital imaging and communication in medicine (DICOM) data could be obtained from the hospital digital storage database. Cases that were suitable for surgical management were confirmed on histology collected during the operation or via biopsy. All hypervascular HCC and FNH were selected by a radiologist over 10 years' experience, with access to relevant clinical information, including the patients' final diagnosis. The radiologist was instructed to qualitatively evaluate whether the hepatic lesion was hypervascular or not. Hypervascular lesions were defined as those of high density, compared with the surrounding liver parenchyma in CT hepatic arteriography.

Nine patients were identified as meeting the inclusion criteria. Six patients (three men and three women, mean age 63 years old) had lesions diagnosed as hypervascular HCC. One of the six patients with HCC had two lesions, 
and the larger lesion was chosen for analysis. Three patients (two men and one woman, mean age 36 years old) had lesions diagnosed as FNH. Two hypervascular HCC were diagnosed on histology collected surgically and the others were diagnosed on radiological findings $(8,15,16)$. Radiological diagnostic criteria of HCC were as follows, arterial hypervascularity and portal or venous phase washout in intravenous dynamic CT. Additionally, the following radiological finding in CT hepatic arteriography supported the diagnosis of HCC; arterial enhancement followed by corona enhancement (17). One FNH was diagnosed on histology collected surgically and two by biopsy histology. The mean diameters of hypervascular HCCs and FNH were $50.3 \pm 27.2$ and $27.3 \pm 3.8 \mathrm{~mm}$ respectively. Lesion diameter was measured using the resected specimen or plain T1-weighted or T2-weighted imaging. There was a single lesion with hemorrhagic content and no lesions with calcification.

\section{DSC-MRI and CTHA}

Angiography assisted CT was performed with a combined 16 detector multi-detector-row CT and digital angiography system (Advantx Light Speed; GE Medical Systems, Milwaukee, WI, USA). After CT during arterial portography (CTAP) and CT hepatic arteriography (CTHA), coeliac, superior mesenteric and common hepatic arteriography were performed. The catheter was subsequently advanced into the proper hepatic, right or left hepatic artery, because the excluding the effect of portal perfusion was important. In case of short or absent proper hepatic artery, the catheter was placed stability at right or left hepatic artery. SL-CTHA was performed with the infusion of $3 \mathrm{~mL}$ of contrast media (Iomeprol, Eizai, Tokyo, Japan; $350 \mathrm{mgI} / \mathrm{mL}$ ) at a rate of $1 \mathrm{~mL} / \mathrm{s}$ in the proper, right or left hepatic artery using a power injector. Two slices at the center of the target lesion were chosen. Scanning at a rate of 0.8 second per rotation began immediately before injection of contrast media. A 40 second continuous scanning technique [auto $\mathrm{mA}(\max 440 \mathrm{~mA})$, $120 \mathrm{kVp}$ ] without table feed was used to obtain two sections with a thickness of $10 \mathrm{~mm}$, beam collimation of $20 \mathrm{~mm}$ and field of view $22-25 \mathrm{~cm}$. Patients were instructed to hold their breath as long as possible during scanning. The images were sorted at each section and observed in both cine mode and manual mode.

MR examinations were acquired with a $1.5 \mathrm{~T}$ superconducting MR unit (Avant, Siemens, Erlangen, Germany). After T1-weighted and T2-weighted images were obtained,
DSC-MRI was performed. Bolus injectable ferucarbotran (Resovist $^{\circledR}$, Bayer, Osaka, Japan) was used. This contrast medium is approved for clinical use in liver MRI in Japan. A total dose of $1.4 \mathrm{~mL}$ (concentration: $27.875 \mathrm{mg} \mathrm{Fe} / \mathrm{mL}$ ) of ferucarbotran was used per patient. Because the dose of ferucarbotran was very low, it could not be placed in the power injector and instead was pooled in an elongation tube and flushed at a rate of $2 \mathrm{~mL} / \mathrm{s}$ with $40 \mathrm{~mL}$ of physiological saline using a power injector.

DSC-MR images were acquired with the echo-planar imaging (EPI) method. For the initial five patients the scan parameters were as follows: TR 1,150 ms; TE $20 \mathrm{~ms}$; FA $70^{\circ}$, EPI factor 154 ; matrix $192 \times 154$; bandwidth $1,628 \mathrm{~Hz}$ per pixel; field of view (FOV) $280-350 \mathrm{~mm}$; slice thickness $7 \mathrm{~mm}$. Fat saturation and parallel imaging was achieved with the generalized auto-calibrating partially parallel acquisition (GRAPPA) algorithm with an acceleration factor (iPAT factor) of 2. Fifteen-slice acquisitions were obtained before administration of contrast media, 2 seconds after the start of administration and thereafter at a temporal resolution of 1.2 seconds over a breath-hold of over 20 seconds. In the latter four patients the scan parameters were changed to improve the time resolution; six-slice acquisitions were obtained per-contrast, 2 seconds after and thereafter at a temporal resolution of 0.46 seconds. The scan parameters were as follows: TR $460 \mathrm{~ms}$; TE $20 \mathrm{~ms}$; FA $90^{\circ}$; matrix $192 \times 156$. The other parameters remained unchanged.

\section{Post processing}

Post-processing was performed by one of the authors using the software PMI 0.4 (18). Concentrations were calculated as the relative signal enhancement $-\ln (\mathrm{S} / \mathrm{S} 0)(19)$, where $\mathrm{S}$ is the postcontrast signal intensity and $\mathrm{S} 0$ the pre-contrast signal intensity. A fixed hematocrit of $45 \%$ was assumed to convert arterial and venous blood concentrations into plasma concentrations.

For DSC-MRI images, arterial input function (AIF) regions were defined semiautomatically over five slices inside the lumen of the abdominal aorta at the same level of the shown hepatic hilum. Selected pixel values on the five slices were averaged to produce the AIF. For each patient a plasma flow map was calculated to aid region of interest (ROI) selection (20,21). The calculation was performed based on the algorithm described in (22): an impulse response function is derived by deconvolving the tissue concentration of every pixel with the AIF using truncated singular value decomposition (TSVD) and a fixed cutoff 
for the singular values at $15 \%$ of the maximum singular value; an estimate of the plasma flow is then derived as the maximum of the impulse response function. Whole lesion regions were selected manually and confirmed by a senior radiologist with over 10 years' experience.

In CTA examinations the AIF regions were defined semiautomatically over the two available slices inside the largest feeding artery available. Estimated plasma flow maps were again calculated (calculating concentration as S-S0, where $\mathrm{S}$ is the post-contrast signal intensity and $\mathrm{S} 0$ the precontrast signal intensity) for each patient and selected pixel values on the two slices were averaged to produce the AIF. Whole lesion regions were selected manually and confirmed by a senior radiologist with over 10 years' experience.

For both CT and MRI modalities, a model-free analysis method was used to derive ROI-based perfusion parameters as described by Sourbron et al. (23). Plasma flow, distribution volume (DV) of contrast agent and estimated mean transit time (EMTT) were estimated with this method.

\section{Statistical analysis}

Correlation between DSC-MRI and SL-CTHA for each perfusion parameter was calculated using Spearman's rank correlation coefficient. The differences between DSCMRI and SL-CTHA for each perfusion parameters were evaluated with Wilcoxon signed-rank test. The differences in each perfusion parameter between hypervascular HCC and FNH were evaluated with Mann-Whitney's U test. A P value less than 0.05 was considered to indicate a statistically significant difference.

\section{Results}

The DSC-MRI and SL-CTHA images of the representative case are shown in Figure 1.

Correlation coefficients and significance values between DSC-MRI and SL-CTHA for each parameter are shown in Figure 2. A strong correlation was obtained with plasma flow $(\mathrm{r}=0.8231, \mathrm{P}=0.0064$, Figure $2 A)$.

No significant correlation was obtained for DV and EMTT (Figure 2B,C). No significant differences were observed between SL-CTHA and DSC-MRI in plasma flow and DV in all subjects (Table 1), but a significant difference was observed for EMTT $(\mathrm{P}=0.04)$. All perfusion parameters showed no significant difference between SL-CTHA and DSC-MRI in FNH. On the other hand, in HCC, DV and EMTT showed significant differences $(\mathrm{P}=0.046$ and 0.046$)$, and plasma flow showed no significant difference (Table 2).

Significant differences in plasma flow between hypervascular HCC and FNH were shown in both SL-CTHA $(\mathrm{P}=0.027)$ and DSC-MRI $(\mathrm{P}=0.00058)$ (Table 3). No significant difference in DV and EMTT between hypervascular HCC and FNH was shown for either SL-CTHA or DSC-MRI.

\section{Discussion}

We focused the possibility of quantitative evaluation in pure blood perfusion excluding extracellular extravascular space. Recently, antiangiogenic therapy or its combined therapy is developed. Gahramanov et al. (11) reported that cerebral blood volume obtained by iron oxide nanoparticle provide the antiangiogenic therapeutic effect more precisely than that by gadolinium-based contrast media. Our final aim is that DSC-MRI derived DV use as a biomarker for antiangiogenic therapy or its combined therapy.

Quantitative values obtained for plasma flow with DSCMRI using SPIO correlated with SL-CTHA. This finding suggests DSC-MRI may be a suitable alternative to SLCTHA in the evaluation of hepatic lesions, consistent with previous non-quantitative studies comparing diagnostic performance of both modalities in the detection of hepatic lesions $(12,13)$. Patients may require repeat examinations to follow up suspicious lesions and compared to SL-CTHA, DSC-MRI is less invasive and does not expose the patient to ionising radiation.

We suggest that DV in DSC-MRI was more accurate than in SL-CTHA. Our assumption was extracellular contrast media perfuses arteries, tumor sinusoids and extracellular-extravascular-interstitial space equivalent to space of Disse in normal liver parenchyma both in HCC and FNH. On the other hand, SPIO perfuses only arteries and tumor sinusoids both in HCC and FNH. The following formula stands among perfusion parameters, DV = plasma flow $\times$ EMTT. EMTT of HCC was significantly different between SL-CTHA and DSC-MRI, while that of FNH was not significantly different. EMTT of FNH was shorter than that of HCC in SL-CTHA, although no significant difference. We suggest that pathological structure influenced the result. FNH has fibrous septa and central scar, and these pathological components are extracellular extravascular space. Therefore, extracellular contrast media leaks and EMTT becomes shorten (24). However, SPIO does not leak from intravascular space during first circulation (25), and thus SPIO is more suitable for evaluating pure tissue perfusion at short durations (26). 
A

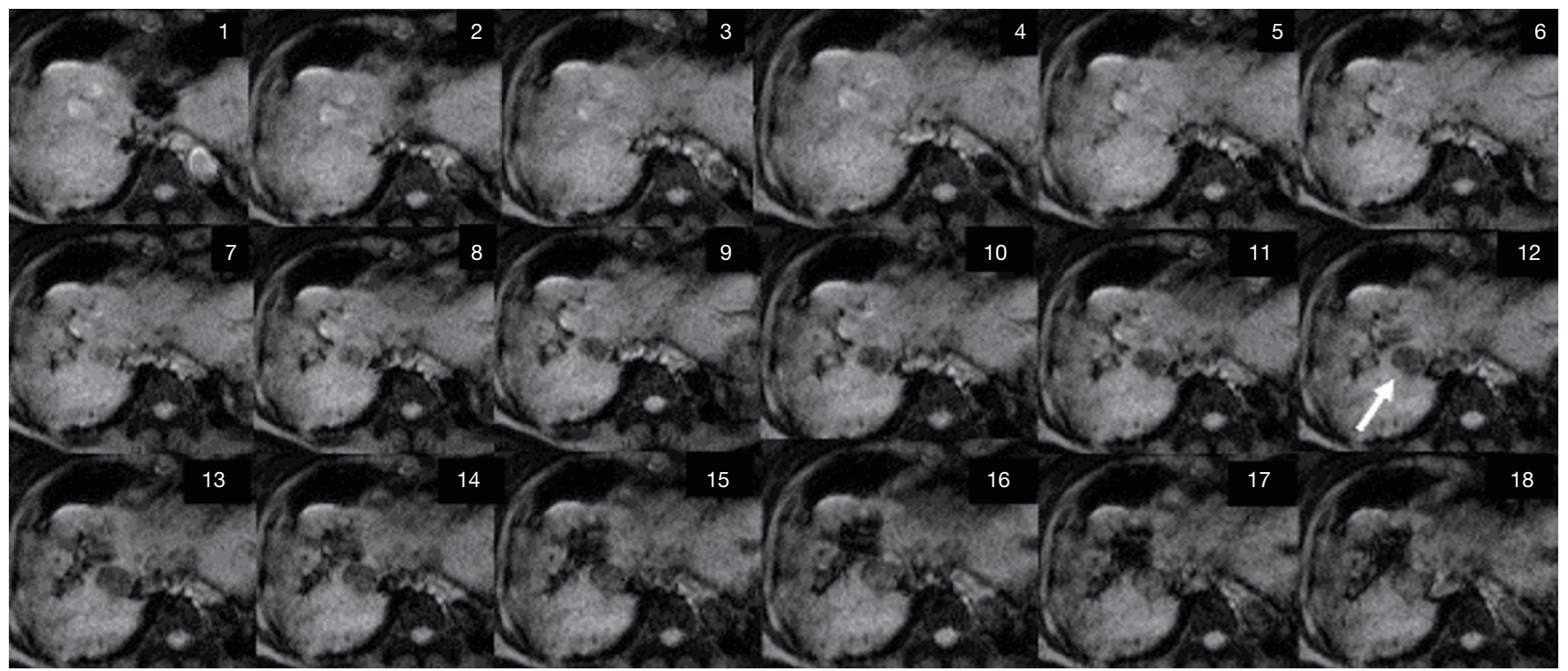

B

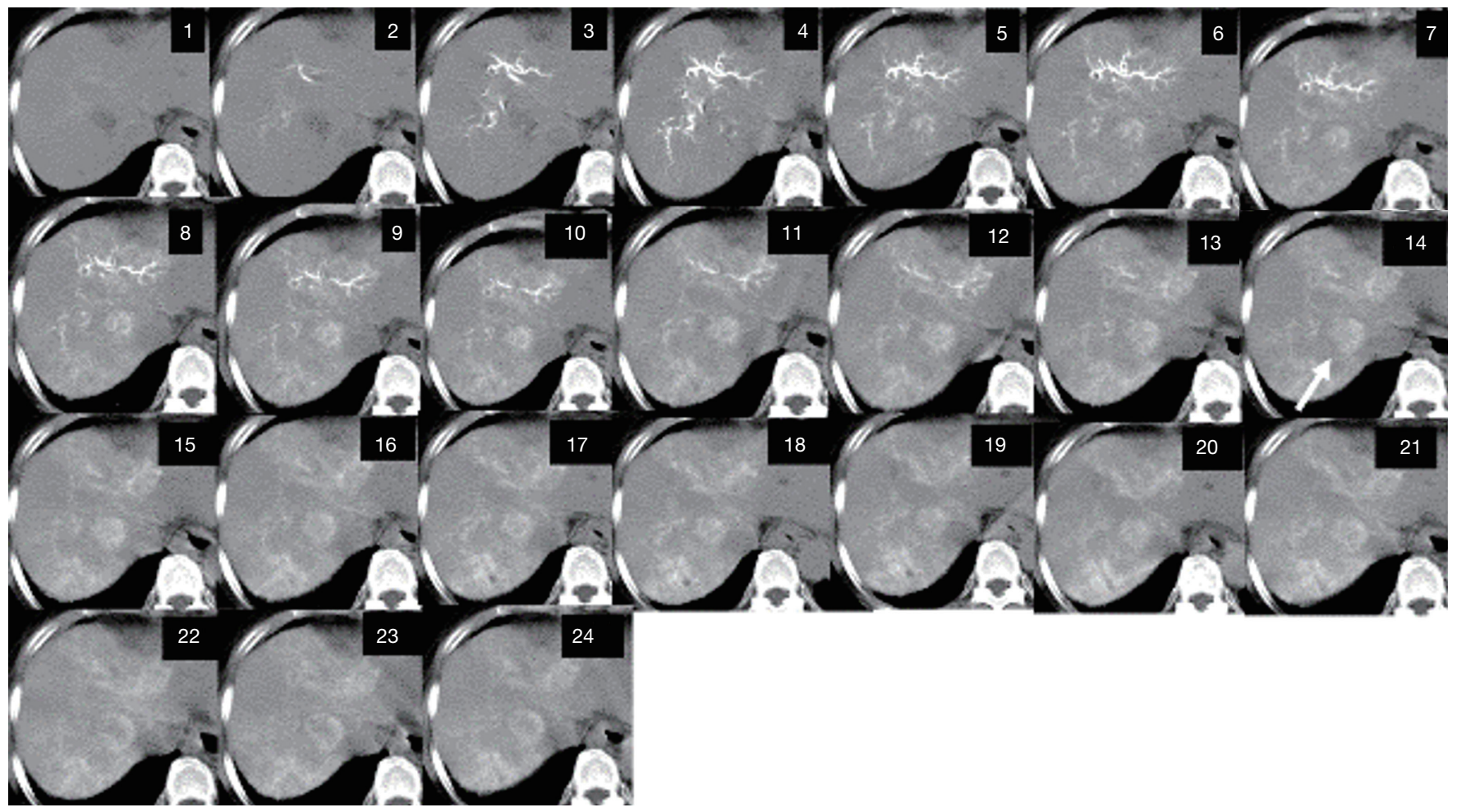

Figure 1 Images in a 73-year-old woman with hepatocellular carcinoma. (A) DSC-MRI shows hypervascular HCC in segment 1. Images were obtained at a temporal resolution of 1.2 seconds over a breath-hold of over 20 seconds. The signal of HCC dropped and recover accompanied surrounding signal drop. The surrounding signal drop indicates corona enhancement. Arrow indicates HCC. (B) CTHA shows hypervascular HCC accompanied corona enhancement in segment 1. Images were obtained at a temporal resolution of 0.8 seconds. Arrow indicates HCC. DSC-MRI, dynamic susceptibility contrast MR imaging; HCC, hepatocellular carcinoma; CTHA, CT hepatic arteriography. 

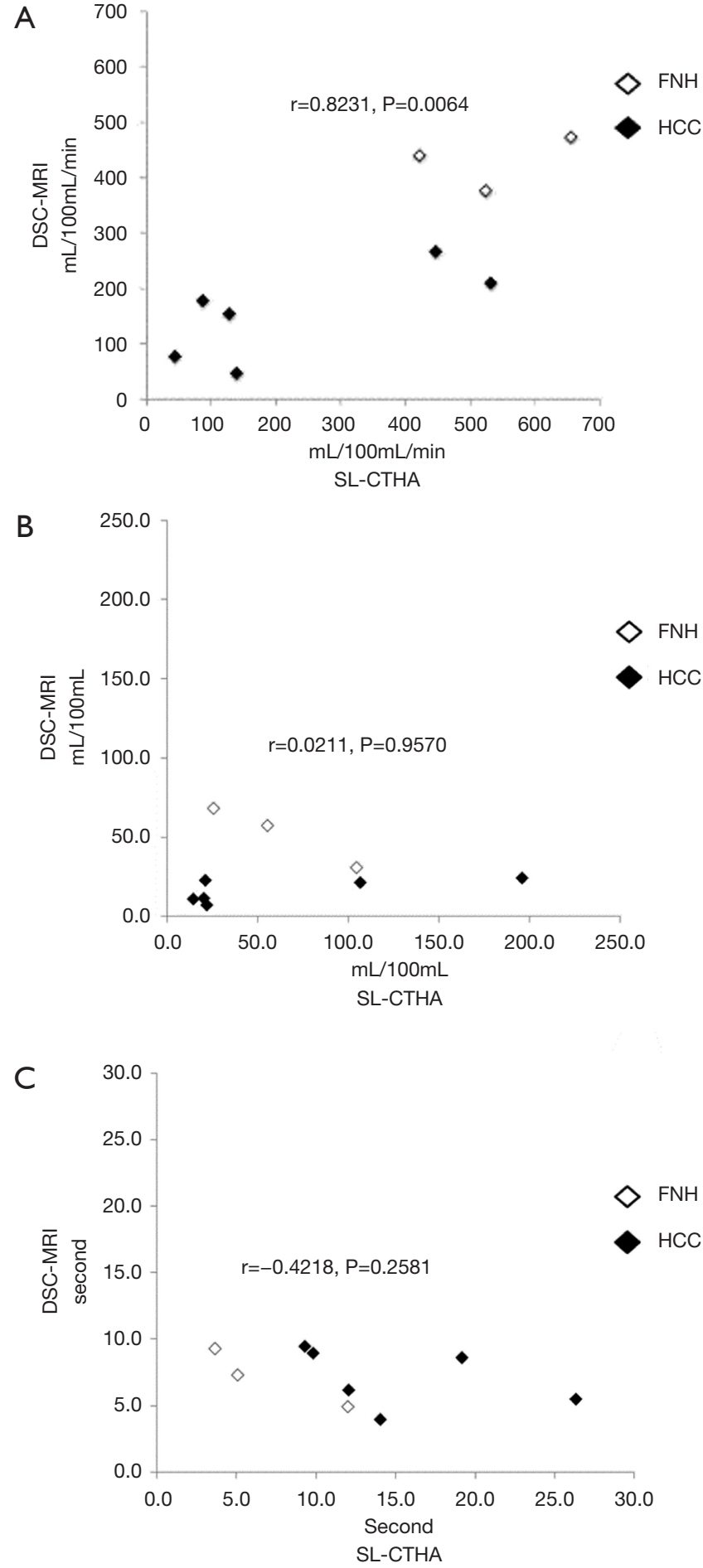

Figure 2 Graph of (A) plasma flow values (mL/100 mL/min), (B) distribution volume (DV) $(\mathrm{mL} / 100 \mathrm{~mL})$ and $(\mathrm{C})$ estimated mean transit time (EMTT) (second) from DCE-MRI and SL-CTHA for each patient. DSC-MRI, dynamic susceptibility contrast MR imaging; SL-CTHA, single level CT during hepatic arteriography; HCC, hepatocellular carcinoma; FNH, focal nodular hyperplasia.
Table 1 Comparing the perfusion parameters between SL-CTHA and DSC-MRI in all cases

\begin{tabular}{lccc}
\hline & $\begin{array}{c}\text { Plasma flow } \\
(\mathrm{mL} / 100 \mathrm{~mL} / \mathrm{min})\end{array}$ & $\begin{array}{c}\text { DV } \\
(\mathrm{mL} / 100 \mathrm{~mL})\end{array}$ & EMTT $(\mathrm{s})$ \\
\hline SL-CTHA & $331.3 \pm 229.9$ & $63.0 \pm 61.7$ & $12.4 \pm 7.0$ \\
DSC-MRI & $245.9 \pm 153.7$ & $28.3 \pm 21.0$ & $7.1 \pm 2.1$ \\
P value & 0.546 & 0.297 & $0.04^{*}$ \\
\hline
\end{tabular}

*, $\mathrm{P}<0.05$. SL-CTHA, single level CT during hepatic arteriography; DSC-MRI, dynamic susceptibility contrast MR imaging; DV, distribution volume; EMTT, estimated mean transit time.

Values for plasma flow were significantly higher in FNH lesions than hyper-vascular HCC with both imaging modalities. This is consistent with previous reports indicating average flow rates in $\mathrm{FNH}$ lesions are higher due to increased vascularity $(16,27)$. Future work will investigate DSC-MRI with SPIO contrast for differentiating FNH and HCC in a larger patient population than included in this study.

This study has several limitations. This study was limited by the small number of patients. This pilot study reveals the possibility of quantitative analysis of liver tumor using SPIO, allowing future work to build on the findings with larger patient cohorts. No gold standard was available for use in all patients: surgery or biopsy for histology was not appropriate in all HCC lesions. Instead we adopted a pragmatic standard for diagnosis and several cases were instead confirmed based on imaging only. Images were collected retrospectively and as a result the image parameters were not consistent in all patients as the hospital routine changed during the period of data collection. Excessive image noise can lead to inaccurate calculations. This may be from difficulties in breathholding, although this is less problematic here since a short acquisition sequence was used, allowing almost all patients to comply with the breath-holding requirements. Magnetic inhomogeneity may lead to inaccurate signal intensities, and also have the potential to cause inaccuracies. The lesion with hemorrhagic content may influence the results of this study due to the susceptibility effect of hemosiderin. This study included one HCC with hemorrhagic content. However, as the hemorrhagic content was small compared to the entire lesion, we believe the influence was relatively small.

In conclusion, this pilot study demonstrates the possibility of quantitative analysis of liver tumor using SPIO and highlights the potential for SPIO in more 
Table 2 Comparing the perfusion parameters between SL-CTHA and DSC-MRI in FNH and HCC

\begin{tabular}{|c|c|c|c|c|c|c|}
\hline & \multicolumn{2}{|c|}{ Plasma flow (mL/100 mL/min) } & \multicolumn{2}{|c|}{$\mathrm{DV}(\mathrm{mL} / 100 \mathrm{~mL})$} & \multicolumn{2}{|c|}{ EMTT (s) } \\
\hline SL-CTHA & $533.8 \pm 95.5$ & $230.0 \pm 187.5$ & $62.0 \pm 33.0$ & $63.4 \pm 67.4$ & $6.9 \pm 3.6$ & $15.1 \pm 6.0$ \\
\hline DSC-MRI & $428.7 \pm 40.0$ & $154.5 \pm 75.0$ & $52.1 \pm 15.7$ & $16.4 \pm 6.6$ & $7.2 \pm 1.8$ & $7.1 \pm 2.0$ \\
\hline$P$ value & 0.285 & 0.345 & 1.00 & $0.046^{*}$ & 1.00 & $0.046^{*}$ \\
\hline
\end{tabular}

*, P<0.05. SL-CTHA, single level CT during hepatic arteriography; DSC-MRI, dynamic susceptibility contrast MR imaging; DV, distribution volume; EMTT, estimated mean transit time; HCC, hepatocellular carcinoma; FNH, focal nodular hyperplasia.

Table 3 Comparing the perfusion parameters of HCC with those of FNH

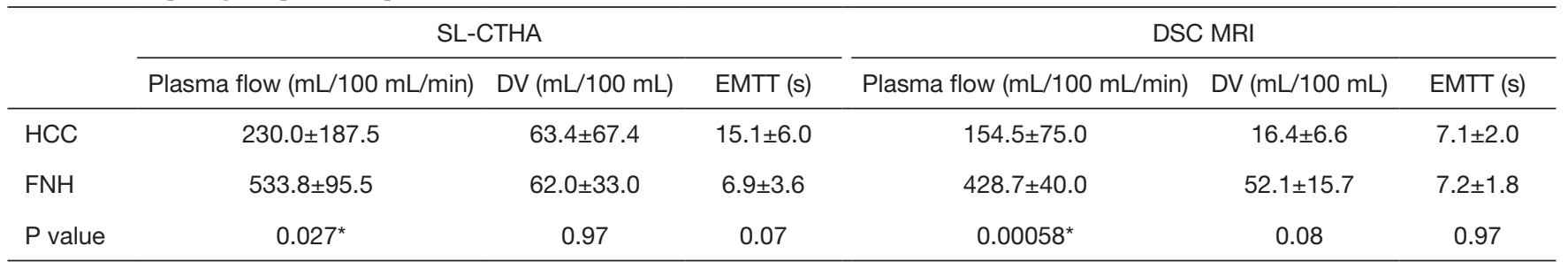

*, $\mathrm{P}<0.05$. SL-CTHA, single level CT during hepatic arteriography; DSC-MRI, dynamic susceptibility contrast MR imaging; DV, distribution volume; EMTT, estimated mean transit time; HCC, hepatocellular carcinoma; FNH, focal nodular hyperplasia.

precisely assessing the perfusion characteristic of hypervascular liver tumors than by using extracellular contrast media. Model free deconvolution based on the algorithm described in (28) with a single arterial input as first-pass method for measuring blood flow and volume in hepatic lesions in DSC-MRI may be a suitable alternative for diagnosis of hepatic lesions. SPIO may be useful in assessing neovascularization of hepatic tumors. Future work may enable to assess the DSC-MRI derived DV use as a biomarker for anti-angiogenic therapy or its combined therapy.

\section{Acknowledgments}

Funding: Tokyo Medical University Cancer Research Foundation.

\section{Footnote}

Conflicts of Interest: All authors have completed the ICMJE uniform disclosure form (available at http://dx.doi. org/10.21037/qims-18-233). KS reports personal fees from Bayer, grants and personal fees from Eisai, grants and personal fees from Daiichi Sankyo, grants and personal fees from FUJIFILM Toyama Chemical, personal fees from Siemens, grants and personal fees from Nihon Medi- physics, outside the submitted work. The other authors have no conflicts of interest to declare.

Ethical Statement: This retrospective study was institutional review board approved and data was collected with implied consent.

Open Access Statement: This is an Open Access article distributed in accordance with the Creative Commons Attribution-NonCommercial-NoDerivs 4.0 International License (CC BY-NC-ND 4.0), which permits the noncommercial replication and distribution of the article with the strict proviso that no changes or edits are made and the original work is properly cited (including links to both the formal publication through the relevant DOI and the license). See: https://creativecommons.org/licenses/by-nc-nd/4.0/.

\section{References}

1. Sourbron S, Sommer WH, Reiser MF, Zech CJ.

Combined Quantification of Liver Perfusion and Function with Dynamic Gadoxetic Acid-enhanced MR Imaging. Radiology 2012;263:874-83.

2. Chen YW, Pan HB, Tseng HH, Hung YT, Huang JS, Chou CP. Assessment of blood flow in hepatocellular carcinoma: correlations of computed tomography 
perfusion imaging and circulating angiogenic factors. Int J Mol Sci 2013;14:17536-52.

3. Taouli B, Johnson RS, Hajdu CH, Oei MT, Merad M, Yee H, Rusinek H. Hepatocellular carcinoma: perfusion quantification with dynamic contrast-enhanced MRI. AJR Am J Roentgenol 2013;201:795-800.

4. Ippolito D, Capraro C, Casiraghi A, Cestari C, Sironi S. Quantitative assessment of tumour associated neovascularisation in patients with liver cirrhosis and hepatocellular carcinoma: role of dynamic-CT perfusion imaging. Eur Radiol 2012;22:803-11.

5. Yamashita Y, Mitsuzaki K, Yi T, Ogata I, Nishiharu T, Urata J, Takahashi M. Small hepatocellular carcinoma in patients with chronic liver damage: prospective comparison of detection with dynamic MR imaging and helical CT of the whole liver. Radiology 1996;200:79-84.

6. Itoh K, Nishimura K, Togashi K, Fujisawa I, Noma S, Minami S, Sagoh T, Nakano Y, Itoh H, Mori K, Hepatocellular carcinoma: MR imaging. Radiology 1987;164:21-5.

7. Fischer MA, Raptis DA, Donati OF, Hunziker R, Schade E, Sotiropoulos GC, McCall J, Bartlett A, Bachellier P, Frilling A, Breitenstein S, Clavien PA, Alkadhi H, Patak MA. MR imaging features for improved diagnosis of hepatocellular carcinoma in the non-cirrhotic liver: Multicenter evaluation. Eur J Radiol 2015;84:1879-87.

8. Sannier A, Cazejust J, Lequoy M, Cervera P, Scatton O, Rosmorduc O, Wendum D. Liver biopsy for diagnosis of presumed benign hepatocellular lesions lacking magnetic resonance imaging diagnostic features of focal nodular hyperplasia. Liver Int 2016;36:1668-76.

9. Amemiya S, Akahane M, Aoki S, Abe O, Kamada K, Saito $\mathrm{N}$, Ohtomo K. Dynamic contrast-enhanced perfusion MR imaging with SPIO: a pilot study. Invest Radiol 2009;44:503-8.

10. Zhang SX, Chen WB, Liang CH, Huang B. Evaluation of the applicability of Resovist in DSC-MR perfusionweighted imaging of rat hyperacute cerebral infarction. Turk Neurosurg 2014;24:344-50.

11. Gahramanov S, Muldoon LL, Li X, Neuwelt EA. Improved perfusion MR imaging assessment of intracerebral tumor blood volume and antiangiogenic therapy efficacy in a rat model with ferumoxytol. Radiology 2011;261:796-804.

12. Saito K, Sugimoto K, Nishio R, Araki Y, Moriyasu F, Kakizaki D, Tokuuye K. Perfusion study of liver lesions with superparamagnetic iron oxide: distinguishing hepatocellular carcinoma from focal nodular hyperplasia.
Clin Imaging 2009;33:447-53.

13. Ichikawa T, Arbab AS, Araki T, Touyama K, Haradome H, Hachiya J, Yamaguchi M, Kumagai H, Aoki S. Perfusion MR imaging with a superparamagnetic iron oxide using T2-weighted and susceptibility-sensitive echoplanar sequences: evaluation of tumor vascularity in hepatocellular carcinoma. AJR Am J Roentgenol 1999;173:207-13.

14. Watanabe S, Katada Y, Gohkyu M, Nakajima M, Kawabata H, Nozaki M. Liver perfusion CT during hepatic arteriography for the hepatocellular carcinoma: dose reduction and quantitative evaluation for normal- and ultralow-dose protocol. Eur J Radiol 2012;81:3993-7.

15. European Association For The Study Of The Liver; European Organisation For Research And Treatment Of Cancer. EASL-EORTC clinical practice guidelines: management of hepatocellular carcinoma. J Hepatol 2012;56:908-43.

16. Ruppert-Kohlmayr AJ, Uggowitzer MM, Kugler C, Zebedin D, Schaffler G, Ruppert GS. Focal nodular hyperplasia and hepatocellular adenoma of the liver: differentiation with multiphasic helical CT. AJR Am J Roentgenol 2001;176:1493-8.

17. Ueda K, Matsui O, Kawamori Y, Nakanuma Y, Kadoya M, Yoshikawa J, Gabata T, Nonomura A, Takashima T. Hypervascular hepatocellular carcinoma: evaluation of hemodynamics with dynamic CT during hepatic arteriography. Radiology 1998;206:161-6.

18. Sourbron S, Ingrisch M, Siefert A, Reiser M, Herrmann K. Quantification of cerebral blood flow, cerebral blood volume, and blood-brain-barrier leakage with DCE-MRI. Magn Reson Med 2009;62:205-17.

19. Do RK, Rusinek H, Taouli B. Dynamic contrastenhanced MR imaging of the liver: current status and future directions. Magn Reson Imaging Clin N Am 2009;17:339-49.

20. Ryeom HK, Kim SH, Kim JY, Kim HJ, Lee JM, Chang YM, Kim YS, Kang DS. Quantitative evaluation of liver function with MRI Using Gd-EOB-DTPA. Korean J Radiol 2004;5:231-9.

21. Nilsson H, Nordell A, Vargas R, Douglas L, Jonas E, Blomqvist L. Assessment of hepatic extraction fraction and input relative blood flow using dynamic hepatocytespecific contrast-enhanced MRI. J Magn Reson Imaging 2009;29:1323-31.

22. Ostergaard L, Sorensen AG, Kwong KK, Weisskoff RM, Gyldensted C, Rosen BR. High resolution measurement of cerebral blood flow using intravascular tracer bolus passages. Part II: Experimental comparison and 
preliminary results. Magn Reson Med 1996;36:726-36.

23. Sourbron S, Dujardin M, Makkat S, Luypaert R. Pixel-bypixel deconvolution of bolus-tracking data: optimization and implementation. Phys Med Biol 2007;52:429-47.

24. Lee JM, Yoon JH, Kim KW. Diagnosis of hepatocellular carcinoma: newer radiological tools. Semin Oncol 2012;39:399-409.

25. Murase K, Assanai P, Takata H, Matsumoto N, Saito S, Nishiura $M$. Kinetic analysis of superparamagnetic iron oxide nanoparticles in the liver of body-temperaturecontrolled mice using dynamic susceptibility contrast magnetic resonance imaging and an empirical mathematical model. Magn Reson Imaging 2015;33:600-10.

Cite this article as: Saito K, Ledsam J, Sourbron S, Araki Y. Validation study of perfusion parameter in hypervascular hepatocellular carcinoma and focal nodular hyperplasia using dynamic susceptibility magnetic resonance imaging with superparamagnetic iron oxide: comparison with single level dynamic CT arteriography. Quant Imaging Med Surg 2020;10(6):1298-1306. doi: 10.21037/qims-18-233
26. Cuenod CA, Balvay D. Perfusion and vascular permeability: basic concepts and measurement in DCE-CT and DCE-MRI. Diagn Interv Imaging 2013;94:1187-204.

27. Marin D, Iannaccone R, Laghi A, Catalano C, Murakami T, Hori M, Kim T, Passariello R. Focal nodular hyperplasia: intraindividual comparison of dynamic gadobenate dimeglumine- and ferucarbotran-enhanced magnetic resonance imaging. J Magn Reson Imaging 2007;25:775-82.

28. Sourbron S, Luypaert R, Morhard D, Seelos K, Reiser M, Peller M. Deconvolution of bolus-tracking data: a comparison of discretization methods. Phys Med Biol 2007;52:6761-78. 